\title{
13 STIMULATION OF THE CYTOSOLIC DSRNA SENSOR MDA-5 INDUCES CELL DEATH IN FIBROBLAST-LIKE SYNOVIOCYTES
}

Olga N Karpus, ${ }^{1}$ Paul P Tak, ${ }^{2}$ Jörg Hamann' 1 Department of Experimental Immunology, Academic Medical Center, University of Amsterdam, Amsterdam, The Netherlands, ${ }^{2}$ Department of Clinical Immunology and Rheumatology, Academic Medical Center, University of Amsterdam, Amsterdam, The Netherlands

10.1136/annrheumdis-2011-201238.13

Background and objectives Rheumatoid arthritis is characterised by hyperplasia of the synovial tissue due to local proliferation of stromal and recruitment of inflammatory immune cells. Accumulation of synovial fibroblasts likely is due to an imbalance between cell proliferation, survival and death. Virus RNA receptors can initiate pro-apoptotic signalling in a variety of cell types. Based on our recent observation that fibroblast-like synoviocytes (FLS) are equipped with functional endosomal (TLR3) and cytosolic (MDA5 and RIGI) sensors for double-stranded (ds)RNA, the authors have studied the consequences of stimulation with dsRNA on the survival of FLS.

Materials and methods FLS from arthritis patients were stimulated with extracellular poly(I:C) or poly(I:C)/3pRNA complexed with PEI for intracellular uptake. Activation of TLR3, MDA5 and RIG-I was confirmed by semi-quantitative 
PCR of response genes. Cell death of FLS after triggering of dsRNA sensors was detected by FACS analysis (annexin V/ PI staining) and MTT assay. mRNA level of 46 genes involved in apoptosis was tested with a multiplex ligation-dependent probe amplification (MLPA) assay. Changes in protein expression were confirmed by Western blot analysis.

Results At concentrations $>0.01 \mu \mathrm{g} / \mathrm{ml}$, stimulation of MDA5 by intracellular delivery of poly(I:C) caused massive cell death in up to $75 \%$ of cultured FLS. In contrast, transfection of $3 p R N A$ triggering RIG-I had a much smaller effect on cell viability, and extracellular administration of the TLR3 ligand poly(I:C) induced death only at the high concentration of $100 \mu \mathrm{g} / \mathrm{ml}$. Type I IFNs did not kill FLS, indicating that cell death was not secondary to IFN receptor signaling. MLPA and biochemical analysis demonstrated a strong induction of the BH3-only pro-apoptotic proteins Puma and Noxa upon dsRNA triggering but was not restricted to MDA5 signaling, suggesting the existence of additional regulatory mechanisms. Treatment of cells with the pan-caspase inhibitor QVD reduced cell death by about $50 \%$, indicating that MDA5-induced cell death in FLS may involve also caspase-independent mechanisms.

Conclusions Triggering of the endosomal dsRNA sensor MDA5 induces expression of pro-apoptotic Puma and Noxa and causes death in cultured FLS. Ligation of MDA5 might be a therapeutic target to overcome apoptosis resistance of FLS in rheumatoid arthritis. 